\title{
The Correlation Between The Treatment Time and Efficacy of Suspended Moxibustion on The Guanyuan Point for The Treatment of Primary Dysmenorrhea Yong-chun $\mathrm{HOU}^{1}$, Zi YAN ${ }^{2}$, Yao-kun XIONG ${ }^{1,{ }^{*},}$, Yan-ting WAN ${ }^{3}$ and
Zhi-yong LIU ${ }^{1,{ }^{*}}$ \\ 1Jiangxi University of Traditional Chinese Medicine, Nanchang 330004, China. \\ ${ }^{2}$ Department of Cadre ward, the fourth Affiliated Hospital of Nanchang University, Nanchang 330002, China. \\ 3Jiangxi People's Armed Police Corps Hospital, Nanchang 330001, China.. 56264931@qq.com(Y.K. Xiong), 469241547@qq.com(Z.Y. Liu). \\ "Corresponding authors
}

Keywords: Guanyuan Point, Primary Dysmenorrhea, Moxibustion, Moxibustion Treatment Time.

\begin{abstract}
Primary dysmenorrhea refers to lower abdominal pain, mild lumbago and discomfort in the lower abdomen during or before the menstrual period. Moxibustion has shown efficacy in the treatment of dysmenorrhea by removing blood stasis and relieving pain. The objective of the current study was to compare the efficacies of different treatment times of suspended moxibustion on the Guanyuan point for the treatment of primary dysmenorrhea of the cold and damp stagnation type to seek the best length of moxibustion treatment. In this study, one hundred and twenty subjects were enrolled in this study and randomly divided into treatment groups $(n=30$ per group): group 1 had suspended moxibustion for $15 \mathrm{~min}$, group 2 had suspended moxibustion for $30 \mathrm{~min}$, group 3 had suspended moxibustion for $60 \mathrm{~min}$ and group 4 had suspended moxibustion for $90 \mathrm{~min}$. The simplified McGill Pain Questionnaire (MPQ) and the COX Menstrual Symptom Scale (CMSS) were used to record results from subjects. Data were analyzed via single factor analysis of variance and the differences between the groups were compared.With regards to the symptom score, significant differences were noted between groups 1,2 and $3(\mathrm{P}<0.01)$, with a treatment time of $60 \mathrm{~min}$ resulting in improved symptoms, while there were no significant differences between groups 3 and 4. The results are as follows: suspended moxibustion on the Guanyuan point for the treatment of primary dysmenorrhea resulted in remarkable improvement of symptoms. The moxibustion treatment time was an important factor that affected the efficacy. A treatment time of 60 min was found to be the optimum length of moxibustion, while more than $60 \mathrm{~min}$ did not further improve symptoms.
\end{abstract}

\section{Introduction}

Primary dysmenorrhea (PD) refers to lower abdominal pain, mild lumbago and discomfort or a feeling of straining in the lower abdomen during or before the menstrual period in the absence of organic pathologically changes. PD generally affects adolescent girls or young women 2 to 3 years after menarche and it is one of the most common gynecological problems. The literature suggests that the incidence is about 30 to $80 \%$ in China [1] and other studies have reported a worldwide incidence of about 20 to $90 \%$ [2-4]. It can have a significant impact on the quality of learning of 
those in education and the work and social lives of young women.

Doctors practicing Western medicine mainly use the oral contraceptive, nonsteroidal anti-inflammatory drugs or calcium channel blockers in the treatment of PD; however, such treatments only alleviate the condition and tend not to offer a permanent cure. Moxibustion has the function of warming meridians, dispelling cold and dampness, removing blood stasis and relieving pain. In the treatment of PD that has been classified as the cold and damp stagnation type, moxibustion has been shown to have significant efficacy. Through observing the effects of different moxibustion treatment times applied via suspended moxibustion to the "Guanyuan" point on PD, this study analyzed the correlation between moxibustion treatment time and the effect on PD symptoms, and explored the optimum treatment time for treating PD.

\section{Data and Methods}

\section{Source of Cases}

In this study, the source of cases was 120 gynecology patients receiving acupuncture and moxibustion at Jiangxi Province Hospital of Traditional Chinese Medicine. Young nulliparous women aged 16 to 30 were selected and the shortest disease course was 6 months, with the longest being 16 years.

\section{Case Selection}

\section{Diagnostic Criteria}

Western medicine diagnostic criteria: according to Obstetrics and Gynecology [5], PD is more common in adolescence and often occurs 1 2 years after menarche; pain often occurs after menstrual onset, but may first appear 12 hours before menstruation, with the most intense pain occurring during the first day of menstruation. It typically lasts 2 3 days and then the pain gradually eases. The pain mainly takes the form of a spasmodic pain in the side-bone area of the lower abdomen, although it may spread to the lumbosacral portion and femoribus internus. The pain may be accompanied by nausea, vomiting, diarrhea, dizziness, fatigue and other symptoms, and serious symptoms include a pale complexion and cold sweating. Gynecological examination finds no abnormalities.

TCM diagnostic criteria: according to the Guiding Principle of Clinical Research on New Traditional Chinese Medicine [6], the diagnostic criteria for dysmenorrhea are as follows: the main symptom is periodic abdominal pain during or before (within 1 week) the menstrual period and it may be accompanied by other discomfort that affects work and life.

Differentiation of TCM symptom types: according to Acupuncture and Moxibustion Therapeutics (7th edition) [7], PD may take the form of the cold-damp coagulation subtype, which manifests as lower abdominal cold pain after or before the onset of the menstrual period. Warming the lower abdomen soothes the pain. Accompanying symptoms may include less menstrual blood, dark almost purple blood with clots, cold limbs, clear and copious urine, white tongue fur and a thready or tight pulse.

\section{Inclusion Criteria.}

1. The patient's symptoms had to be in accordance with the diagnosis standard of PD.

2. PD was of the stagnation subtype caused by cold and damp. 3. Only nulliparous women aged 16 30 who were willing to accept treatment and be included in the 
research project were enrolled. 4. Subjects had regular menstruation (28 \pm 7 days). 5 . The disease course was from 6 months to 16 years. 6. Subjects had not taken other drugs to treat their PD and no other methods of treating PD had been taken for nearly a month. 7. Patients were conscious, with normal behavior and clear speech, and could correctly detect the moxibustion sensation.

\section{Exclusion Criteria}

1. Patients with severe primary diseases, such as diseases of the cardiovascular system, liver, kidney and hematopoietic system, and mental health patients were excluded. 2. Patients that, according to the estimation of researchers, were in environments that could impact on the quality of the data were excluded, such as those with frequent environment changes and those in unstable living environments, who could easily be lost during follow up. 3. Patients who fainted after moxibustion or rejected moxibustion as a treatment option were excluded.

\section{Elimination Criteria.}

1. Misdiagnosis or false acceptance. 2. Patients without treatment records or incomplete information affecting the judgment of efficacy. 3. Those with other conditions affecting treatment during the course of moxibustion. 4. Patients using other, self-administered treatment methods during the treatment or patients that provided incomplete information, making efficacy difficult to determine.

\section{Intervention Measure}

Suspended moxibustion was administered at the Guanyuan point by first circling the moxa to warm the local blood passing it along the pathways of the channels to dredge them, including the collateral channels. "Bird-pecking" moxibustion was then used to increase meridian Qi and, finally, warming moxibustion was used to strengthen the impact of the treatment. Starting from 5 days prior to the onset of pain, treatment was provided once a day for 7 days for each menstrual cycle, with a total of three treatment courses. Follow up was arranged after three menstrual cycles.

\section{Grouping Method}

One hundred and twenty subjects were randomly divided into four groups as follows. Treatment group 1 had suspended moxibustion for $15 \mathrm{~min}$, group 2 for $30 \mathrm{~min}$, group 3 for $60 \mathrm{~min}$ and group 4 for $90 \mathrm{~min}$.

\section{Outcome Index}

\section{Dysmenorrhea Score Sheet}

The score sheet was prepared based on a marked scale of dysmenorrhea symptoms and the grading standard of pain degree under the Guiding Principle of Clinical Research on New Traditional Chinese Medicine [6]. According to the standard, dysmenorrhea can be divided into three degrees:

1.Severe: symptoms during or before the menstrual period, comprising lower abdomen pain that is hard to endure, trouble sitting comfortably or sleeping, pain that seriously affects work, study and daily life, and a need to stay in bed, accompanied by an aching pain at the waist, a pale complexion, cold, dripping sweat, cold limbs, vomiting, diarrhea or a straining feeling towards the anus. Pain-relieving measures have no significant effect. This resulted in a dysmenorrhea symptom score of above 14 points.

2.Moderate: symptoms during or before the menstrual period, comprising lower 
abdomen pain that is hard to endure, accompanied by an aching pain at the waist, nausea, vomiting and cold limbs. Pain-relieving measures provide relief. This resulted in a dysmenorrhea symptom score of 8-13.5 points.

3.Mild: symptoms during or before the menstrual period, comprising obvious lower abdomen pain, accompanied by an aching pain at the waist. However, the patient is still able to work, with no systemic symptoms, although they may need to occasionally take painkillers. This resulted in a dysmenorrhea symptom score of below 8 points.

\section{Simplified Mcgill Pain Questionnaire (MPQ)}

The pain questionnaire [8] was divided into three parts:

1.Pain rating index (PRI): eleven sensory words and four emotional words. According to the records filled in by the patients, the PRI feelings, emotion scores and total scores were calculated. According to the total score, the pain scale was divided into four levels - none, mild, moderate and severe, which were counted as $0,1,2$ and 3 points, respectively.

2.Visual analogue scale (VAS): pain recorded on a scale over 24 hours to give an average value. Patients used a $10 \mathrm{~cm}$ long VAS scale, marked with digits from 0-10. Subjects were asked to mark their pain in the appropriate position between the scales. Points were then counted as follows: 0 to $2 \mathrm{~cm}$, painless, counted as 0 points; 3 to 5 $\mathrm{cm}$, mild pain, counted as 2 points; 6 to $8 \mathrm{~cm}$, moderate pain, counted as 4 points; 9 to $10 \mathrm{~cm}$, severe pain, counted as 6 points.

3.Present pain intensity (PPI): according to the pain degree, pain intensity was divided into six degrees-painless, mild discomfort, discomfort, uncomfortable, terrible pain and extremely painful, which were counted as $0,1,2,3,4$ and 5 points, respectively.

\section{Cox Dysmenorrhea Symptom Scale (Cmss)}

CMSS [9] was used to evaluate the pain frequency and pain degree of dysmenorrhea symptoms and was composed of 18 items. Each item was divided into levels between zero and three, which were recorded as $0,1,2$ and 3 points, respectively.

\section{Statistical Analysis}

SPSS 21 statistical software was used to analyze the data of the four groups with single factor variance and to compare the differences among the four groups. When the variance was homogeneous, least significant difference (LSD) was used, and when the variance was not homogeneous, Tamhane's T2 was used to make pairwise comparisons. The chi-squared test $\left(\chi^{2}\right)$ was used to compare the categorical data of the four groups or Fisher's exact probability test. The $\mathrm{CMH} \chi^{2}$ test and the rank-sum test were used to compare the efficacies in the rank data.

\section{Experimental Results}

\section{Comparison of Baseline Data}

\section{Comparison of Age And Disease Course of The Four Groups}

Treatment group 1: the minimum age was 17 , the maximum age was 29 , the shortest disease course was 6 months and the longest disease course was 15 years. Treatment group 2: the minimum age was 16, the maximum age was 30 , the shortest disease course was 8 months and the longest disease course was 14 years. Treatment group 3: the minimum age was 16 , the maximum age was 28 , the shortest disease course was 9 
months and the longest disease course was 16 years. Treatment group 4: the minimum age was 18 , the maximum age was 30 , the shortest disease course was 7 months and the longest disease course was 15 years. After the t-test, the study found that there were no significant differences with regards to age and disease course among the four groups $(\mathrm{P}>0.05$; see Table 1$)$.

Table 1 Comparison of the ages and disease courses of the four groups

\begin{tabular}{cccc}
\hline Group & $N$ & Average age (years)* & $\begin{array}{c}\text { Average disease } \\
\text { course (years)* }\end{array}$ \\
\hline Treatment group 1 & 30 & $23.27 \pm 5.61$ & $7.15 \pm 2.81$ \\
Treatment group 2 & 30 & $23.04 \pm 5.19$ & $6.87 \pm 2.72$ \\
Treatment group 3 & 30 & $22.61 \pm 5.76$ & $6.93 \pm 2.64$ \\
Treatment group 4 & 30 & $23.72 \pm 6.03$ & $6.27 \pm 2.34$ \\
\hline
\end{tabular}

$* \mathrm{P}>0.05$.

\section{Comparison of Disease Distribution of The Four Groups}

Treatment group 1:7 mild cases, 15 moderate cases, 8 severe cases.

Treatment group 2: 6 mild cases, 15 moderate cases, 9 severe cases.

Treatment group 3: 8 mild cases, 16 moderate cases, 6 severe cases.

Treatment group 4: 9 mild cases, 14 moderate cases, 7 severe cases.

After the chi-squared test, the study found that there were no significant differences with regards to the disease state among the four groups $(\mathrm{P}>0.05)$ and the extent of PD was comparable among the groups (see Table 2).

Table 2 Comparison of the disease distribution of the four groups

\begin{tabular}{ccccc}
\hline Group & $N$ & Mild* & Moderate* & Severe* \\
\hline Treatment group 1 & 30 & 7 & 15 & 8 \\
Treatment group 2 & 30 & 6 & 15 & 9 \\
Treatment group 3 & 30 & 8 & 16 & 6 \\
Treatment group 4 & 30 & 9 & 14 & 7 \\
\hline
\end{tabular}

\section{Comparison of The Symptom Scores of The Four Groups}

There were no significant differences between the symptom scores among the four groups before treatment $(\mathrm{P}>0.05)$. After treatment, there were significant differences between groups 1,2 and $3(\mathrm{P}<0.01)$, showing that the short-term efficacy of $60 \mathrm{~min}$ moxibustion was superior to that of $30 \mathrm{~min}$, while $30 \mathrm{~min}$ was superior to that of 15 min. There were no significant differences between groups 3 and $4(\mathrm{P}>0.05)$, showing that the efficacy of 90 min moxibustion was equivalent to that of $60 \mathrm{~min}$. 


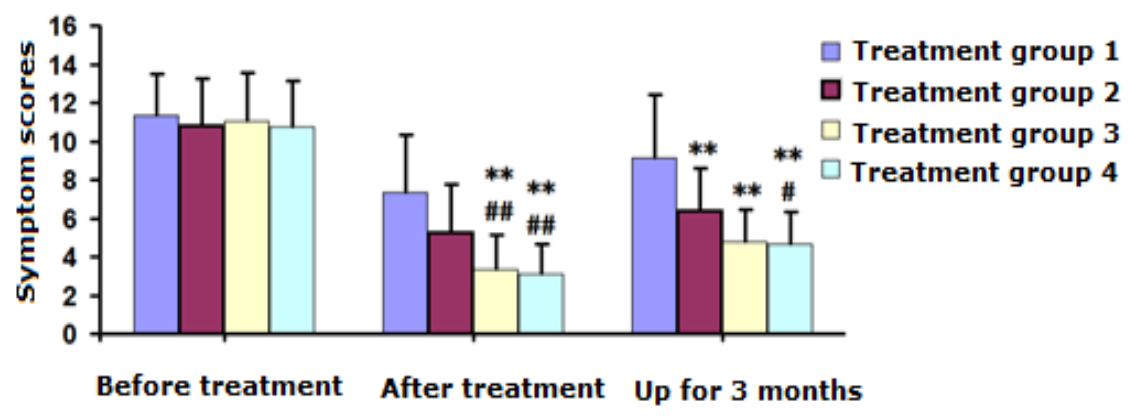

Figure 1 Comparison of the symptom scores of the four groups. Compared with treatment group 1, $* \mathrm{P}<0.05$ and $* * \mathrm{P}<0.01$; compared with treatment group 2 , \#P<0.05 and \#\#P<0.01.

After follow up at 3 months, it was found that there were significant differences ( $\mathrm{P}$ $<0.01$ ) between groups 1,2 and 3 , showing that 60 min moxibustion was significantly better than $30 \mathrm{~min}$ and $15 \mathrm{~min}$ over the treatment course of 3 months. However, the study found that there were no significant differences $(\mathrm{P}>0.05)$ between groups 3 and 4, showing that, with regards to the long-term efficacy, $60 \mathrm{~min}$ moxibustion is as effective as $90 \mathrm{~min}$.

\section{Comparison of The Simplified MPQ Results of The Four Groups}

There were no significant differences in the PRI, VAS and PPI among the four groups before treatment $(\mathrm{P}>0.05)$. After treatment, there were significant differences among groups 1,2 and $3(\mathrm{P}<0.01)$, which showed that improvements on the PRI, VAS and PPI following $60 \mathrm{~min}$ moxibustion were superior to those of $30 \mathrm{~min}$, while the results following $30 \mathrm{~min}$ moxibustion were superior to those of $15 \mathrm{~min}$. There were no significant differences between groups 3 and $4(\mathrm{P}>0.05)$, which showed that the efficacy of $90 \mathrm{~min}$ moxibustion was equivalent to that of 60 min moxibustion.

After follow up at 3 months, the study found that there were significant differences $(\mathrm{P}<0.01)$ among groups 1,2 and 3 , showing that 60 min moxibustion was significantly better than $30 \mathrm{~min}$ and $15 \mathrm{~min}$. Meanwhile, the study found that there were no significant differences $(\mathrm{P}>0.05)$ between treatment groups 3 and $4(\mathrm{P}>0.05)$, showing that, with regards to the long-term efficacy, 60 min moxibustion was as affective as $90 \mathrm{~min}$. 

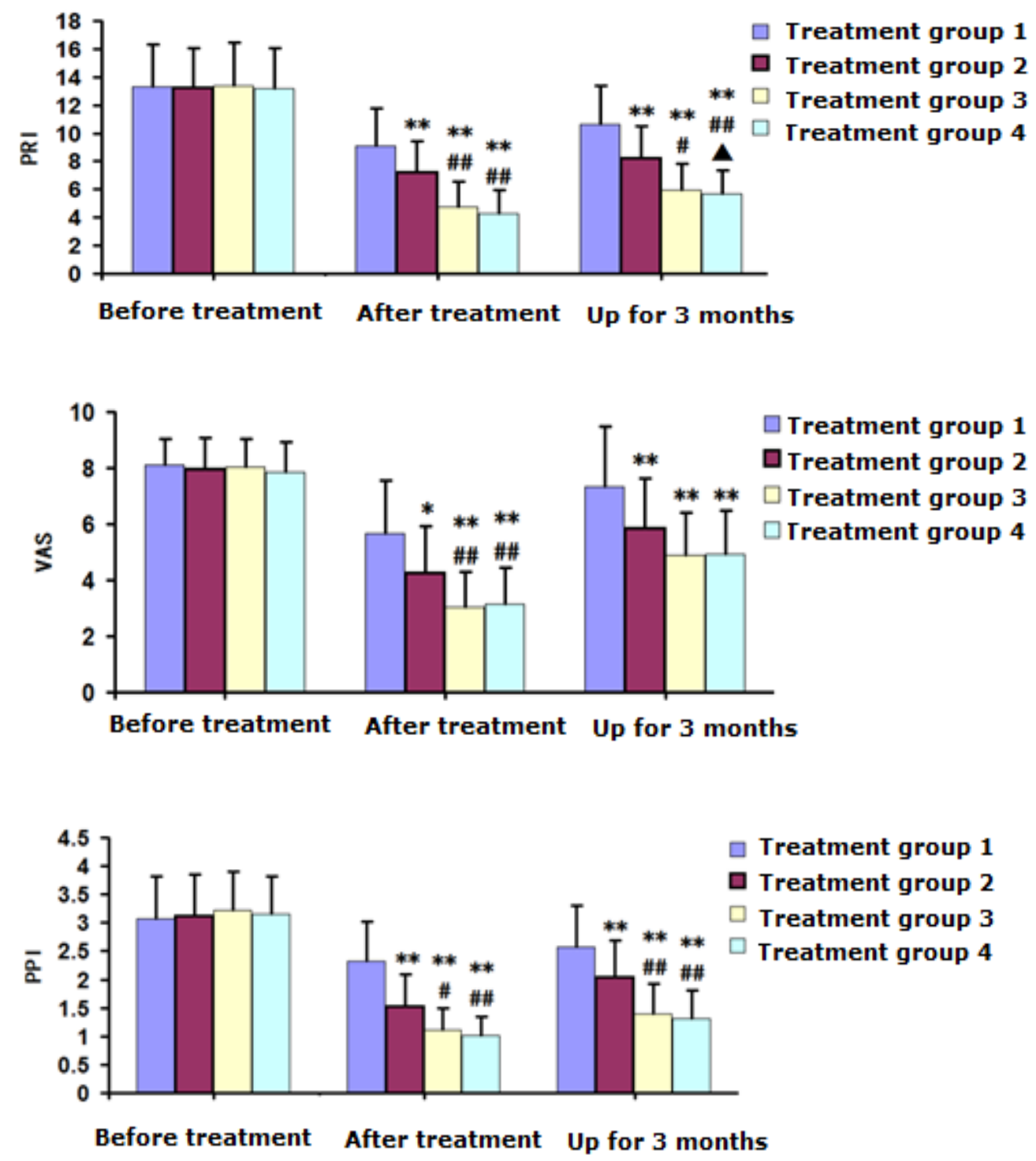

Figure 2 Comparison of the simplified MPQ results of the four groups in terms of PRI, VAS and PPI. Compared with treatment group 1 , $* \mathrm{P}<0.05$ and $* * \mathrm{P}<0.01$; compared with treatment group 2 , \#P $<$ 0.05 and \#\#P $<0.01$; compared with treatment group 3, $\mathbf{\Delta} \mathrm{P}<0.05$.

Comparison of the COX pain scale results of the four groups

There were no significant differences in pain frequency and pain degree among the four groups before treatment $(\mathrm{P}>0.05)$. After treatment, there were significant differences among groups 1,2 and $3(\mathrm{P}<0.01)$, showing that the pain frequency and pain degree improved following $60 \mathrm{~min}$ moxibustion and the improvements were superior to those of $30 \mathrm{~min}$ moxibustion, while $30 \mathrm{~min}$ moxibustion was superior to 15 min moxibustion. There were no significant differences between groups 3 and 4 (P > 0.05), showing that the efficacy of 90 min moxibustion was equivalent to that of 60 min moxibustion.

After follow up at 3 months, the study found that there were significant differences $(\mathrm{P}<0.01)$ among groups 1,2 and 3 , showing that 60 min moxibustion was significantly better than either $30 \mathrm{~min}$ or $15 \mathrm{~min}$. Meanwhile, the study found that there were no significant differences $(\mathrm{P}>0.05)$ between treatment groups 3 and $4(\mathrm{P}>$ $0.05)$, showing that, with regards to the long-term efficacy, 90 min moxibustion was comparable to 60 min moxibustion. 


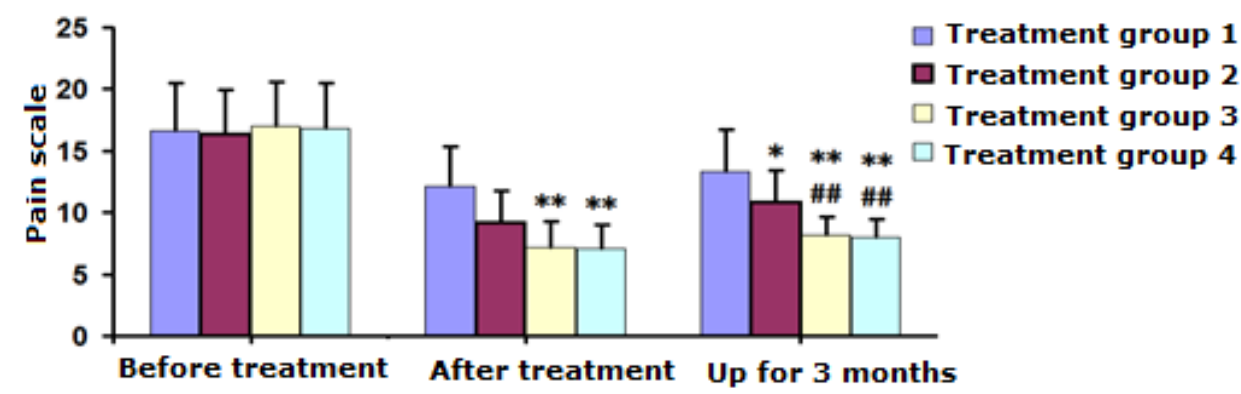

Figure 3 Comparison of the COX pain scale for the four groups. Compared with treatment group 1 , *P $<0.05$ and $* * \mathrm{P}<0.01$; compared with treatment group 2, \#\#P $<0.01$.

\section{Discussion}

Dysmenorrhea can be described as abdominal pain during menstruation and was first recorded in tradition Chinese medicine in the Synopsis of Prescriptions of the Golden Chamber: Symptoms and Cures for Women Diseases, where the following was stated in relation to dysmenorrhea: "morbid leucorrhea, menstrual disorders, lower abdomen pain, reoccurs after a month, treat it with Kwa root so as to disperse it". At present, traditional Chinese medicine believes that dysmenorrhea is caused by internal and external pathogens that lead to the malfunction of Qi-blood, the organs, and the main and collateral channels affecting the uterus. In traditional Chinese medicine, dysmenorrhea is mainly divided into two major types: 1. "when there is inharmoniousness, there is pain" and 2. "when there is stoppage, there is pain". Dysmenorrhea can then be divided into six subtypes: cold and damp stagnation, accumulation of damp heat, Qi stagnation and blood stasis, Qi and blood deficiency, Yang deficiency with cold, and deficiency of the liver and kidneys.

This study selected PD manifesting as cold and damp stagnation for investigation. Jingyue Compendium Women says, "if cold-evil stagnates in main and collateral channels, or the reverse of exogenous cold, or exposes in inadvertent cold in daily life, coagulation occurs, thus leading to pain without deficiency". Fu Qing-zhu's Gynecology says, "cold-dampness is a pathogenic factor". The Chong and Ren meridians in women lie in the lower Jiao and menstrual blood flows from the two meridians. If the Chong and Ren meridians are filled with cold dampness, internal disorder occurs. When the two meridians contend with each other, pain can occur. The pathogenesis of the cold-damp stagnation subtype of PD can be summarized as the following: "cold evil" damages the body due to a raw and cold diet during menstruation, or pathogenic cold dampness coagulates in vivo due to long-term residence in cold, wet places. Thus, blood flow is blocked and dysmenorrhea occurs. Thus, the type of therapeutic principle should warm and activate the meridians, expel cold and dampness, promote blood circulation and relieve pain. So, the best moxibustion therapy for dysmenorrhea is suspended moxibustion to the Guanyuan point.

As the acupoint of the conception channel, the Mu-front acupoint of the small intestine meridian and the convergent acupoint of three Yin channels in the foot, the Guanyuan point may rule syndromes affecting the three Yin channels in the foot, the small intestine and the conception channel. Since ancient times, the Guanyuan point has been used as an important point for tonification. Plain Questions: Pain Theory says, "the vital channel arises from Guanyuan". The above description shows that Guanyuan is connected with the vital channel and the Guanyuan point has the 
function of regulating the vital channel and warming the uterus. The Great Compendium of Acupuncture and Moxibustion says, "Guanyuan, three inches below the navel, the Mu-front acupoint of the small intestine meridian and the convergent acupoint of three Yin channels of the foot and Ren channel...is closely related with the accumulation of pathogenic cold deficiency, gripping pain below the umbilicus that sneaks in the external genital organs". Zhang Jingyue summed up the effect of the Guanyuan point "to treat accumulation of pathogenic cold and various impairments due to deficiencies" and went on to say that "in order to treat cold damage of Yin syndrome and uninhibited copious urine, cold damage, leucorrhea with reddish discharge in women, doctors should apply moxibustion to Guanyuan point". By means of thermal moxibustion, one can warmly nourish the main and collateral channels, tonify Yang-Qi and promote Qi to activate the blood. When applying moxibustion to Guanyuan, diseases are said to heal themselves.

Moxibustion "dose" is determined by three factors: area, strength and time. As the first two factors are affectively fixed, the moxibustion dose is mainly decided by the treatment time. Whether the moxibustion dose is sufficient or not directly affects the clinical efficacy. Therefore, establishing the optimum moxibustion time is the key to improving the clinical efficacy. The purpose of this study was to observe the efficacy differences between PD subjects treated with moxibustion for different lengths of time, with suspended moxibustion applied on the Guanyuan point. It was also of interest to explore the relationship between moxibustion treatment time and efficacy and to find the best moxibustion treatment length for the relief of PD. The results showed that with an increase of moxibustion treatment time the favorable effects of suspended moxibustion on PD increased. There were significant differences between group 3 (suspended moxibustion for $60 \mathrm{~min}$ ) and groups 1 (suspended moxibustion for $15 \mathrm{~min}$ ) and 2 (suspended moxibustion for $30 \mathrm{~min}$ ). Meanwhile, comparing groups 3 and 4 (suspended moxibustion for $90 \mathrm{~min}$ ), there were no significant differences with regards to the dysmenorrhea index, which showed that, for PD patients, $60 \mathrm{~min}$ of suspended moxibustion was optimal. Therefore, $60 \mathrm{~min}$ moxibustion can be considered the best dose since more than $60 \mathrm{~min}$ did not further enhance the efficacy.

In summary, suspended moxibustion on the Guanyuan point can significantly improve the symptoms of PD and 60 min moxibustion was found to be the optimum treatment length.

\section{Acknowledgments}

This research was financially supported by Jiangxi Provincial Department of Education technology projects (Grant NO. GJJ10560), Jiangxi Provincial Science and Technology Department support Project (2014ZBBG70012) and Jiangxi provincial health department foundation of China (2014A022), (2014A096). We are pleased to thank F.Y. XIAO for the English language polishing and F. Li for her excellent assistance in editorial changes.

\section{References}

[1] Collaborative Group for National Women's Menstrual Physical Constants. Investigation and analysis on Chinese women's menstrual physical constants. Chin J Obstet Gynecol. 15(1980)219-220.

[2] Davis AR, Westhoff CL. Primary dysmenorrhea in adolescent girls and treatment with oral contraceptives. J Pediatr Adolesc Gynecol. 14(2001)3-8. 
[3] Banikarim C, Chaeko MR, Kelder SH. Prevalence and impact of dysmenorrheal on Hispanic female adolescents. Arch Pediatr Adolesc Med. 154(2000)1226-1229.

[4] Strinic T, Bukovic D, Pavelic L, et al. Anthropological and clinical characteristics in adolescent women with dysmenorrhea. Coll Antropol, 27(2003)707711 .

[5] Yue Jie. Obstetrics and Gynecology (7th Edition). (People's Health Publishing House, Beijing, 2008)pp. 318-319.

[6] Zheng Xiaoyu. Guiding Principle of Clinical Research on New Traditional Chinese Medicine. (Chinese Medical Science and Technology Press, Beijing, 2002)pp. 345-349.

[7] Wang Qicai. Acupuncture and Moxibustion Therapy. (China Press of Traditional Chinese Medicine, Beijing, 2003)pp. 155-156.

[8] Me1Zaek R. The short form McGill painquestionnaire.Pain,30(1987)191-192.

[9] Cox DJ, Meyer RG. Behavioral treatment parameters With primary dysmenorrhea. J Behav Med,1(1978)297-298. 